\title{
ACCESS TO INFLUENZA VACCINE IN EAST HARLEM AND THE BRONX DURING A NATIONAL VACCINE SHORTAGE
}

\author{
Danielle C. Ompad, PhD; Sandro Galea, MD, DrPH; Shannon Blaney, \\ MPH; Micaela H. Coady, MS; Sarah Sisco, MPH, MSSW; Kathryn Glidden, \\ RN; David Vlahov, PhD
}

\begin{abstract}
In October 2004, one of the major producers of the U.S. influenza vaccine supply announced that their vaccine would not be available because of production problems, resulting in approximately half of the anticipated supply suddenly becoming unavailable. This study was part of a larger effort using community-based participatory research (CBPR) principles to distribute influenza vaccine to hard-to-reach populations. Given the extant literature suggesting economic and racial disparities in influenza vaccine access in times of adequate supply and our inability to distribute vaccine due to the shortage, we sought to examine vaccine access as well as awareness of the vaccine shortage and its impact on health-seeking behaviors in eight racially-diverse and economicallydisadvantaged neighborhoods in New York City (NYC) during the shortage. In our study few people had been vaccinated, both among the general community and among high risk groups; vaccination rates for adults in priority groups and non-priority groups were $21.0 \%$ and $3.5 \%$. Awareness of the 2004 vaccine shortage was widespread with over $90 \%$ being aware of the shortage. While most attributed the shortage to production problems, almost $20 \%$ said that it was due to the government not wanting to make the vaccine available. Many respondents said they would be more likely to seek vaccination during the current and subsequent influenza seasons because of the shortage. The target neighborhoods were significantly affected by the national influenza vaccine shortage. This study highlights the challenges of meeting the preventive health care needs of hard-to-reach populations in times of public health crisis.
\end{abstract}

D. C. Ompad, S. Galea, S. Blaney, M. H. Coady, S. Sisco, K. Glidden, D. Vlahov, Center for Urban Epidemiologic Studies, New York Academy of Medicine, 1216 Fifth Avenue, New York, New York, 10029, USA; S. Galea, Department of Epidemiology, The University of Michigan, School of Public Health, 1214 S. University, Ann Arbor, Michigan, 48104-2548, USA; S. Galea, D. Vlahov, Department of Epidemiology, Columbia University, Mailman School of Public Health, New York, New York, 10032, USA.

Requests for reprints should be addressed to D. C. Ompad, PhD, Center for Urban Epidemiologic Studies, New York Academy of Medicine, 1216 Fifth Avenue, New York, New York, 10029, USA; e-mail: dompad@nyam.org. 
KEY WORDS: influenza; vaccination; vaccine shortage; health disparities; community-based participatory research.

\section{INTRODUCTION}

Influenza is a major public health concern. There were over 61,000 deaths from influenza and pneumonia in the United States in 2004, making these conditions the eighth leading cause of death. ${ }^{1}$ During the most recent influenza epidemics in the U.S., it was estimated that up to 220,000 people were hospitalized due to influenza. ${ }^{2}$ In October 2004, one of the major producers of the U.S. influenza vaccine supply announced that their vaccine would not be available because of production problems. ${ }^{3}$ The effect on the U.S. vaccine supply was unexpected and dramatic, with approximately half of the anticipated supply suddenly becoming unavailable.

A recent study examined influenza vaccination coverage in the U.S. during the 2004-2005 vaccine shortage. ${ }^{4}$ According to this study $42 \%$ of adults in priority groups (e.g., persons aged $\geq 65$ years, health care workers with patient contact, household contacts of children $<6$ months of age and persons with one or more of the following conditions: asthma and other lung problems, heart problems, diabetes, kidney problems, weakened immune system anemia and pregnancy) and $8.8 \%$ of adults not in a priority group received a vaccination between September 2004 and January 2005.

There are well documented racial/ethnic disparities in influenza immunization rates. A study using data from the 1996 Medical Expenditure Panel Survey compared influenza vaccination levels of different racial/ ethnic groups. ${ }^{5}$ Among 2,309 persons aged $\geq 65$, Whites had the highest vaccination coverage $(68.0 \%)$ followed by Hispanics $(61.7 \%)$, and Blacks $(47.3 \%)$. Adjustment for potential confounders including health risk, age distribution, health status, family size, ambulatory care visits, and poverty level failed to account for these differences. In terms of socioeconomic status and social disadvantage, characteristics that have been shown to be associated with lower vaccination rates include living in residence owned by someone else, marital status and unemployment. ${ }^{6}$

Given the extant literature suggesting economic and racial disparities in influenza vaccine access in times of adequate supply, we sought to examine vaccine access in racially-diverse and economically-disadvantaged neighborhoods in New York City during the recent shortage. In addition, we aimed to examine awareness of the vaccine shortage and its impact on health-seeking behaviors. 
Danielle C. Ompad et al. 197

\section{METHODS}

This study was conducted as a part of Project VIVA (Venue-Intensive Vaccination for Adults), which was designed to assess barriers to vaccination in specific communities in East Harlem and the Bronx and to use this research to guide a vaccination intervention in these same neighborhoods. ${ }^{7}$ These two communities are among the most socio-economically disadvantaged communities in New York City. Within East Harlem and the South Bronx, our study was carried out in eight small neighborhoods. These neighborhoods are areas of particular disadvantage within the larger communities. They were defined by a process of consultation with community members from East Harlem and the South Bronx.

From January to December 2004, outreach workers had been in the field conducting surveys in an effort to enumerate the target population and provide educational materials about influenza and influenza vaccine. At the time the vaccine shortage was announced, we were gearing up for the distribution of vaccine for Project VIVA, which had been scheduled to begin in early October 2004. Due to the national shortage, we did not receive vaccine and therefore conducted surveys to assess awareness of the shortage and access to the vaccine in our target neighborhoods.

We aimed to recruit hard-to-reach persons (e.g., active injection drug users, sex workers, undocumented immigrants, and elderly shutins) in each of the eight neighborhoods within East Harlem and the Bronx, New York City. Briefly, to recruit subjects we used venue-based sampling specified through community-based collaboration to find locations where hard-to-reach populations congregate. ${ }^{8}$ The study was approved by the Institutional Review Board of the New York Academy of Medicine.

From October to December 2004, outreach workers approached potential participants and asked them if they would be willing to complete a brief survey. Those providing oral informed consent were administered a brief, anonymous survey about vaccination history and barriers to vaccination. The survey included questions about sociodemographic characteristics, access to health care, and risk behaviors (e.g. drug use, commercial sex work, etc.). In addition, we included a series of questions related to the vaccine shortage, including knowledge of the shortage, access to the vaccine during the shortage, and attitudes towards Project VIVA in light of the shortage. All people engaged by the outreach workers were offered information about influenza vaccine, 
including information of where to get it, regardless of their willingness to participate in the survey.

\section{RESULTS}

We first examined sociodemographic characteristics of the 272 people interviewed during this study period (data not shown). Most $(84.8 \%)$ were aged 49 years or younger, $13.3 \%$ were aged 50 to 64 and $1.9 \%$ were 65 years or older. The population was $75 \%$ male and predominately Hispanic $(69.6 \%)$, followed by Black $(23.3 \%)$, other $(4.1 \%)$ and White $(3.0 \%)$. Most had a total yearly legal income of less than $\$ 9600$ per year $(63.3 \%)$. Almost all considered themselves to be stably housed $(81.2 \%)$, although some were transitionally housed $(15.1 \%)$ or homeless $(3.3 \%)$. Almost $13.0 \%$ had been hungry in the last 6 months. A small proportion had injected drugs or traded sex in the last six months $(3.3 \%$ and $3.7 \%$, respectively).

In terms of access to health and social services, $78.5 \%$ had some type of health insurance (data not shown). Almost $85 \%$ reported having access to routine health care. Among those without routine medical care, the most common reason for not having it was not having enough money. The majority $(72 \%)$ had an influenza shot at least once in their lifetime, of whom $24.1 \%$ were vaccinated in the past year.

Table 1 reports knowledge of the vaccine shortage and its affect on health-seeking behaviors. Most (91.1\%) were aware of the vaccine shortage, having found out through television $(79.0 \%)$, the newspaper $(34.8 \%)$, word-of-mouth $(16.6 \%)$, and/or a doctor or health care provider $(11.7 \%)$. Most attributed the shortage to manufacturer production problems $(59.5 \%) ; 18.9 \%$ said the government did not want to make the vaccine available. More than one-quarter (26.9\%) said the shortage had changed their mind about getting the influenza vaccine this year, of whom $70.8 \%$ said they were now more likely to seek out an influenza shot this year. Slightly fewer $(24.4 \%)$ said the shortage had changed their mind about getting the influenza vaccine next year, of whom $(80.3 \%)$ were more likely to try to seek out the influenza shot. In terms of the impact of the shortage on our relationship with the community, $25.7 \%$ of respondents said the shortage changed their mind about receiving services from Project VIVA, with $86.6 \%$ being more likely to seek out of services.

Overall, $245(90.1 \%)$ people surveyed were medically eligible for an influenza shot. Of those medically eligible, $116(47.7 \%)$ had tried to obtain 


\section{TABLE 1}

Attitudes Towards the U.S. Influenza Vaccine Shortage and Access to Vaccine Among 272 Residents of New York City: October - December 2004

\begin{tabular}{lrr}
\hline Attitudes towards the influenza vaccine shortage & $N$ & $\%$ \\
\hline Aware of national influenza vaccine shortage & 247 & 91.1 \\
Source of news about shortage & 195 & 79.0 \\
Television & 86 & 34.8 \\
Newspaper & 41 & 16.6 \\
Word of mouth & 29 & 11.7 \\
Doctor or health care provider & 8 & 3.2 \\
Hospital or clinic & 1 & 0.4 \\
Dept. of health or other govt. agency & 3 & 1.2 \\
Other & 154 & 59.5 \\
Reason(s) thinks that shortage exists & 49 & 18.9 \\
Production problem w/ manufacturer & 7 & 2.7 \\
Government did not want to make vaccine available & 21 & 8.1 \\
New York City Department of Health decided not necessary & 32 & 12.4 \\
Influenza vaccine limited to parts of country & & \\
Other & &
\end{tabular}

Shortage changed mind about getting an influenza vaccine this season

Yes

No

$191 \quad 70.5$

$\begin{array}{lll}\text { Prefer not to say } & 2 & 0.7\end{array}$

$\begin{array}{lll}\text { Don't know } & 5 & 1.9\end{array}$

How shortage changed mind- this season

$\begin{array}{lll}\text { More likely to seek out an influenza vaccine } & 51 & 70.8\end{array}$

$\begin{array}{lll}\text { Less likely to seek out an influenza vaccine } & 21 & 29.2\end{array}$

Shortage changes mind about getting an influenza vaccine next year

Yes

$66 \quad 24.4$

No

$190 \quad 70.4$

$\begin{array}{lll}\text { Don't know } & 14 & 5.2\end{array}$

How shortage changed mind- next year

$\begin{array}{lll}\text { More likely to seek out an influenza vaccine } & 53 & 80.3\end{array}$

$\begin{array}{lll}\text { Less likely to seek out an influenza vaccine } & 13 & 19.7\end{array}$

Shortage changes mind about receiving services from Project VIVA

Yes

$69 \quad 25.7$

No

$185 \quad 68.8$

$\begin{array}{lrr}\text { Prefer not to say } & 2 & 0.7\end{array}$

\begin{tabular}{lll} 
Don't know & 13 & 4.8 \\
\hline
\end{tabular} 
TABLE 1 (Continued)

\begin{tabular}{lrr}
\hline Attitudes towards the influenza vaccine shortage & $N$ & $\%$ \\
\hline How vaccine shortage changes mind about Project VIVA services & & \\
More likely to seek out Project VIVA services & 58 & 86.6 \\
Less likely to seek out Project VIVA services & 9 & 13.4 \\
Access to influenza vaccination during the shortage & 245 & 90.1 \\
Medically eligible for influenza shot & 116 & 47.7 \\
Has tried to get influenza shot since Oct. 1, 2004 & 26 & 10.6 \\
Received an influenza shot since Oct. 1, 2004 & 100 & 40.8 \\
Member of group at high risk for complications & 64 & 64.0 \\
Has tried to get influenza shot since Oct. 1, 2004 & 21 & 21.0 \\
Received an influenza shot since Oct. 1, 2004 &
\end{tabular}

an influenza shot and $26(10.6 \%)$ had received a vaccination since October 1, 2004. Among the $100(40.8 \%)$ persons in high risk groups for complications from the influenza $64(64.0 \%)$ tried to get vaccinated and 21 $(21.0 \%)$ actually received an influenza shot. Among the 145 in the nonpriority groups $52(35.9 \%)$ tried to get vaccinated and $5(3.5 \%)$ received an influenza shot. There were no significant differences by race in terms of trying to obtain a vaccination; however, those who reported other race were significantly $(p=0.02)$ more likely to have been vaccinated since October 1, 2004 than Hispanics, Blacks or Whites $(40.0 \%$ vs. 9.7\%, 9.8\% and $0 \%$, respectively - data not shown).

\section{DISGUSSION}

We found that awareness of the 2004 vaccine shortage was widespread in eight disadvantaged neighborhoods in NYC with over $90 \%$ of the population being aware of the shortage. This level of knowledge is consistent with a previous study which reported that $92 \%$ of elderly patients surveyed in Pittsburgh were aware of the 2000 influenza vaccine delay. ${ }^{9}$ While most recognized that the shortage was due to production problems, almost $20 \%$ said that the shortage was due to the government not wanting to make the vaccine available. This finding suggests that issues of trust may be important to consider when bringing interventions to ethnically diverse populations.

Many respondents said they would be more likely to seek influenza vaccination during the current and subsequent influenza seasons because 
of the shortage, suggesting that vaccine shortages may increase awareness of the availability and utility of a vaccine. More than half of medically eligible individuals and one-third of those at risk for complications did not try to get the vaccine. One nationally representative telephone survey recently reported that $51 \%$ persons aged 65 or older and $63 \%$ of persons with chronic illnesses did not try to get vaccinated. ${ }^{10}$ A recent review has suggested that interventions that provide vaccine outside medical settings such as primary and tertiary care are needed. ${ }^{11}$ It is unclear why our population was more likely to try to get vaccinated, but it may have been related to outreach efforts we had conducted in the community prior to the influenza season.

In our study few people had been vaccinated, both among the general community and among high risk groups; the vaccination rates for adults in priority groups and non-priority groups were $21.0 \%$ and $3.5 \%$, respectively as compared to national estimates of $42 \%$ and $8.8 \%$. ${ }^{4}$ Thus, compared to estimates from the $\mathrm{CDC}$, overall vaccination rates were lower in this population suggesting that economically-disadvantaged communities would benefit from focused vaccination efforts in times of mass shortage.

The 2004 influenza vaccine shortage resulted in a surprising and dramatic reduction in vaccine supply in the United States. Vaccine supply and distribution in the U.S. is not primarily driven by need, but rather by the costs of research, development, testing and manufacturing and a market that is small compared to other drug products. ${ }^{12}$ This situation has led to maldistribution of vaccine, often leaving sicker and/or poorer patients unprotected. ${ }^{13}$ In this study, we observed that a group of economically-disadvantaged and ethnically-diverse communities in New York City were significantly affected by the national influenza vaccine shortage. This study highlights the challenges of meeting the preventive health care needs of hard-to-reach populations in times of public health crisis. Further study is needed to determine if such disparities exist for similar communities in times of shortage. Directed and culturally appropriate interventions are needed to address the preventive health care needs of these communities.

\section{ACKNOWLEDGEMENTS}

This study was conducted as a part of Project VIVA (Venue-Intensive Vaccination for Adults) and is a collaboration between the Harlem 
Community and Academic Partnership (H-CAP) and the Center for Urban Epidemiologic Studies (CUES). The VIVA Intervention Working Group members are: Ann Boyer (Mt. Sinai Medical Center, Women's Information Network and Birdsong); Robert Brackbill (New York City Department of Health and Mental Hygiene); Brian Brown (deceased, New York Harm Reduction Educators); Jose Caraballo (Palladia, Inc.); Micaela Coady (CUES); Sandro Galea (CUES); Kay Glidden (CUES); Karyn London (Mt. Sinai Medical Center); Gail Love (Women’s Information Network); Pat Monahan (East Harlem Community Health Committee, Inc. and Little Sisters of the Assumption Family Health Service); Danielle Ompad (CUES); Erica Phillips (New York Presbyterian Hospital, Cornell Medical College); Sarah Sisco (CUES); Sharon Stancliff (Harm Reduction Coalition); David Vlahov (CUES), and Linda Weiss (Office of Special Populations at the New York Academy of Medicine). This study was supported by a grant from the National Institute on Drug Abuse (DA017004) and the Merck Foundation.

\section{REFERENGES}

1. Miniño AM, Heron MP, Smith BL. Deaths: Preliminary data for 2004. Natl Vital Stat Rep 2006; 54:150 .

2. Advisory Committee on Immunization Practices. Prevention and control of influenza. MMWR Morb Mortal Wkly Rep 2002; 51:1-31.

3. Pollack A. U.S. Will Miss Half Its Supply Of Flu Vaccine. New York TImes 2004 Oct 6; A1, A20.

4. Estimated influenza vaccination coverage among adults and children-United States, September 1, 2004-January 31, 2005. MMWR Morb Mortal Wkly Rep 2005; 54:304-307.

5. Marin MG, Johanson WG Jr., Salas-Lopez D. Influenza vaccination among minority populations in the United States. Prev Med 2002; 34:235-241.

6. Petersen RL, Saag K, Wallace RB, Doebbeling BN. Influenza and pneumococcal vaccine receipt in older persons with chronic disease: a population-based study. Med Care 1999; 37:502-509.

7. Bryant WK, Ompad DC, Sisco S, Blaney S, Glidden K, Phillips E, et al. Determinants of influenza vaccination in hard-to-reach urban populations. Prev Med 2006; 43:60-70.

8. Muhib FB, Lin LS, Stueve A, Miller RL, Ford WL, Johnson WD, et al. A venue-based method for sampling hard-to-reach populations. Public Health Rep 2001; 116(Suppl 1), 216-222.

9. Santibanez TA, Nowalk MP, Zimmerman RK, Bruehlman RD. Effects of the year 2000 influenza vaccine delay on elderly patients' attitudes and behaviors. Prev Med 2003; 37:417-423.

10. Experiences with obtaining influenza vaccination among persons in priority groups during a vaccine shortage-United States, October-November, 2004. MMWR Morb Mortal Wkly Rep 2004; 53:1153-1155.

11. Ompad DC, Galea S, Vlahov D. Distribution of influenza vaccine to high-risk groups. Epidemiol Rev 2006; 28:54-70.

12. Offit PA. Why are pharmaceutical companies gradually abandoning vaccines? Health Aff (Millwood) 2005; 24:622-630.

13. Wynia MK. Markets and public health: pushing and pulling vaccines into production. Am J Bioeth 2006; 6:3-6. 